\title{
Design for a Image Processing Virtual Simulation Platform
}

\author{
Dan Tian*, Shu-xin Shi and Yong-shuai Li
}

\author{
School of Information Engineering, Shenyang University, Shenyang, 110044, China
}

\begin{abstract}
Keywords: Virtual simulation platform, Image denoising, Feature extraction, Image mosaic, GUI. Abstract. A image processing virtual simulation platform is designed based on MATLAB GUI. This platform has good interactivity and expansibility, which integrates multiple functional modules including basic function module, noise adding module, image denoising module, feature extraction module, and image mosaic module. The platform can help students learning the basic theory and methods for digital image processing.
\end{abstract}

\section{Introduction}

MATLAB GUI ${ }^{[1]}$ (Graphical User Interface) provides a visual interactive platform, which can be used for settling code uniformly. It is relatively easy to get familiar with and requires little knowledge of programming. This paper constructs a image processing virtual simulation platform based on MATLAB GUI. The platform has five modules, which integrate basic function (rotation, brighten, graying, zooming, and restoration), noise adding (impulse noise, Gaussian noise, and multiple noise), image denoising ${ }^{[2]}$ (median filter and linear filter), feature extraction ${ }^{[3]}$ (susan, harris and sift ${ }^{[4]}$ feature detection), and image mosaic ${ }^{[5]}$ functions. We conduct the platform main interface based on $\mathrm{M}$ language. Clicking the arbitrarily submodule in the main interface, the corresponding submodule can be open and the corresponding function can be realized.

\section{Platform Construction}

MATLAB GUI is composed of the window, menu, icon, cursor, keys, dialog boxes, text and other graphical objects. It allows users to customize how to interact with MATLAB. This paper designs a image processing virtual simulation platform based on MATLAB GUI to realize the basic function in digital image processing. Default user interface is shown in Figure.1.

The menu bar is composed of file, algorithms, and clear menu. Click the file menu, we can add the original image 1 and the original image 2, and save the image processing results. Click the algorithms menu, we can select the corresponding image processing function shown in Figure.2. Click the clear menu, the original image window and the processed image window can be restored. The toolbar offers the open, save and zooming functions.

Selecting the basic function module, we can realize the corresponding image processing function, which intergrades image rotation, image brighten, image graying, image zooming, and image restoration functions. Click the corresponding module, we can set the needed parameter. Such as, rotation angular, scaling plateau, etc. Figure. 3 shows the function of the image zooming module, which can capture the region of interest for the image.

Selecting the noise module, we can add kinds of noise with specific intensity, such as salt and pepper noise, Gaussian noise, and multiple noise. Salt and pepper noise is a kind of black and white bright dark spot noise. Gaussian noise is a kind of random noise, whose amplitude obeys Gaussian distribution. When the power spectral density of the Gaussian noise is uniformly distributed, it is named as Gaussian white noise. Multiple noise has the multiplication relationship with the image. Click the corresponding submodule, we can set the noise intensity and add noise. Figure.4 shows the simulation result for adding salt and pepper noise, whose intensity is 0.03 . 


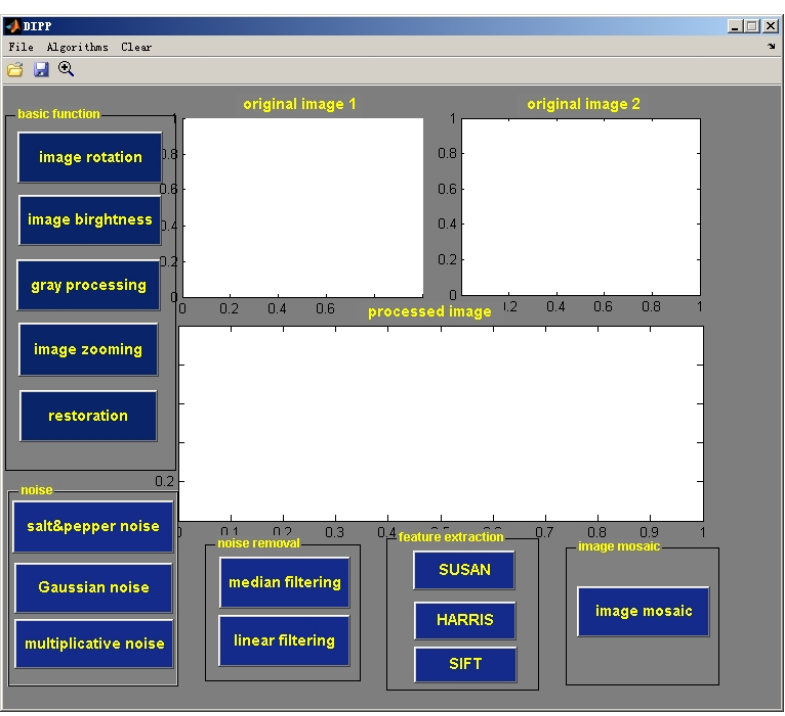

Figure 1. Default user interface

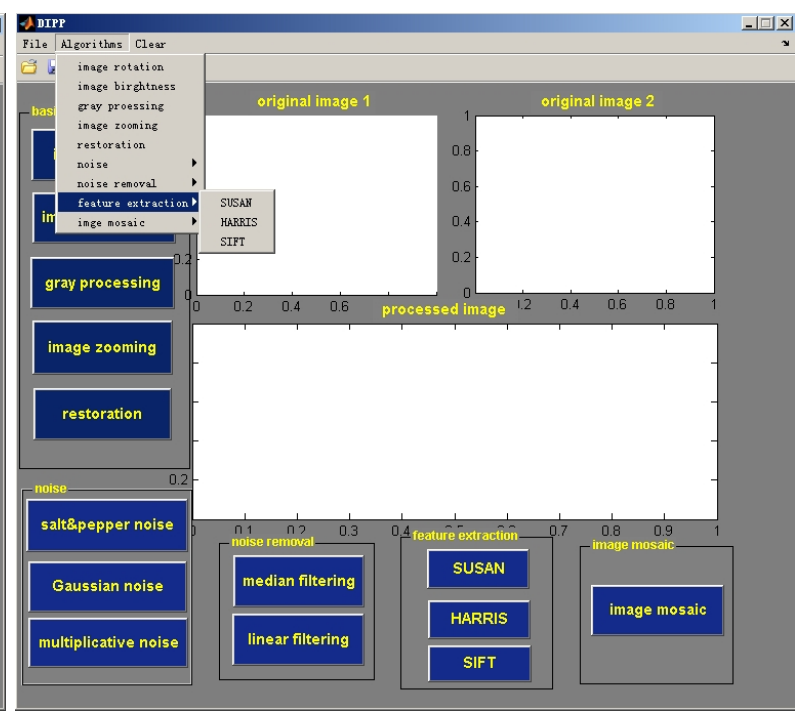

Figure 2. Menu options

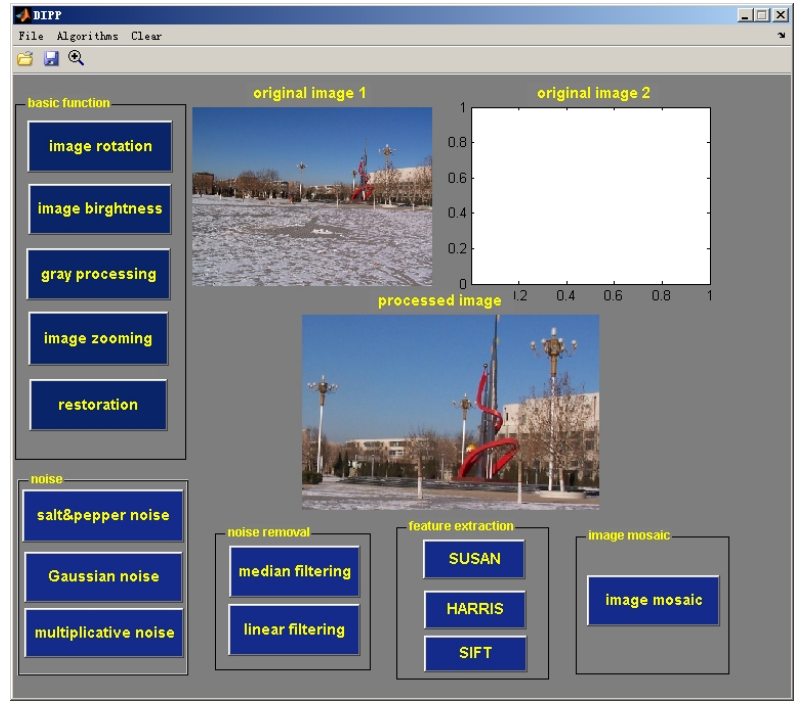

Figure 3. Image zooming module function

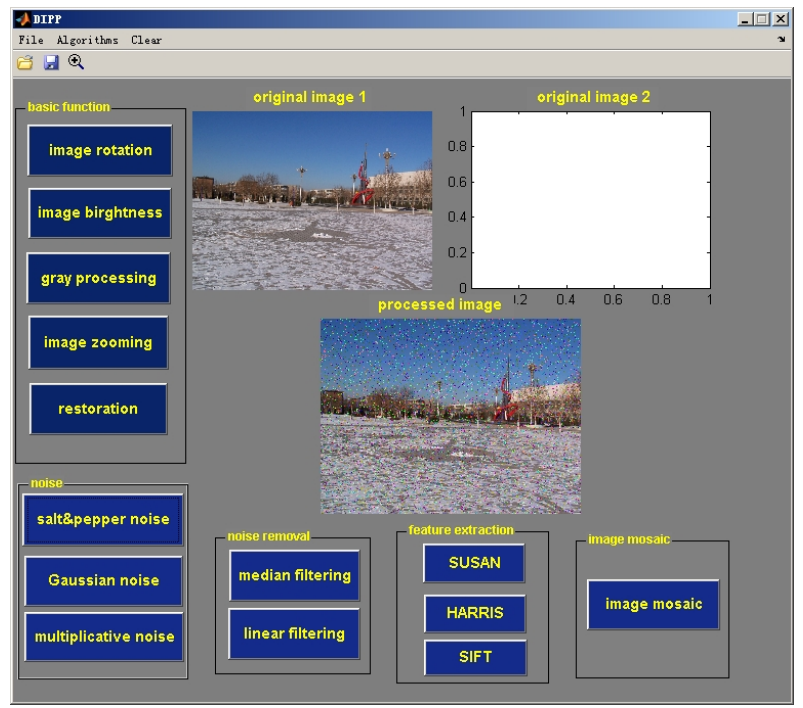

Figure 4. Noise adding module function

Selecting the noise removal module, we can process the image using median filter and linear filter methods respectively. Median filter is a kind of nonlinear smoothing technique. It sets the pixel gray value as the median of all pixels within a certain neighborhood window. Linear filter also processes 
the pixel gray directly, but by a linear smoothing way. Figure.5 shows the simulation results using these two methods for denoising the noise image in Figure.4. Particularly necessary to point out is that the noisy image should be grayed before denoising procssing. Simulation results show that the median filter is more suitable for removing the salt and pepper noise.

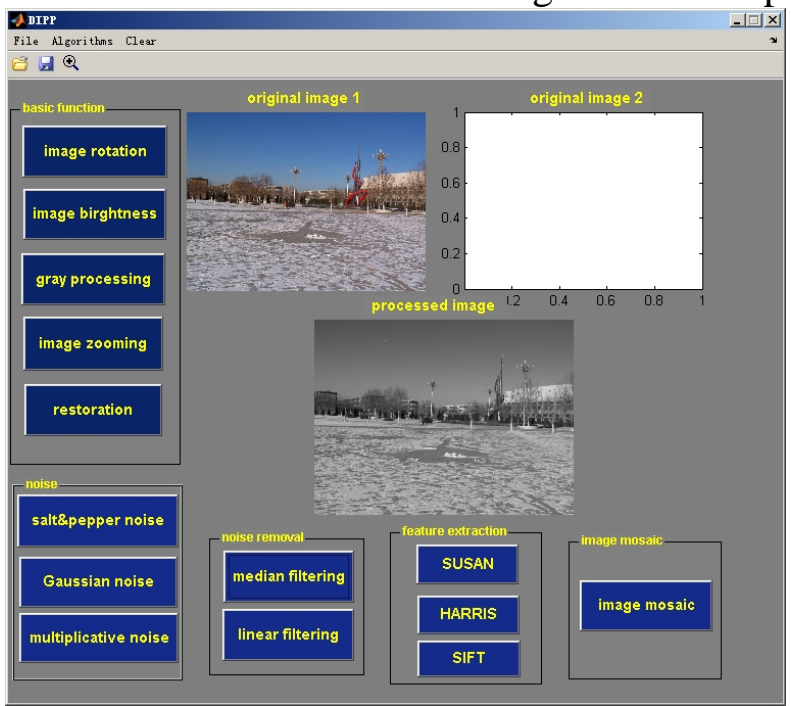

(a) median filter

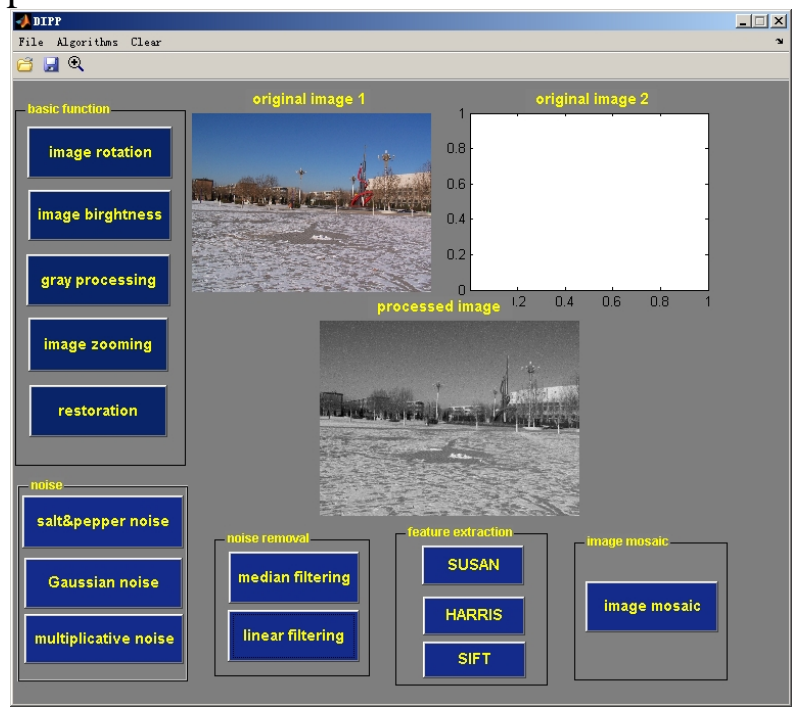

(b) linear filter

Figure5. Image denoising module function

Selecting the feature extraction module, we can capture the feature point based on susan, harris and sift algorithm respectively. Harris is a kind of corner detection operator based on differential operation and autocorrelation matrix operation. Susan corner detection is realized by calculating the corner response function around the template domain. Sift feature detection is realized by founding the extreme point in scale space. Figure.6 shows the feature extraction simulation results using the harris algorithm.

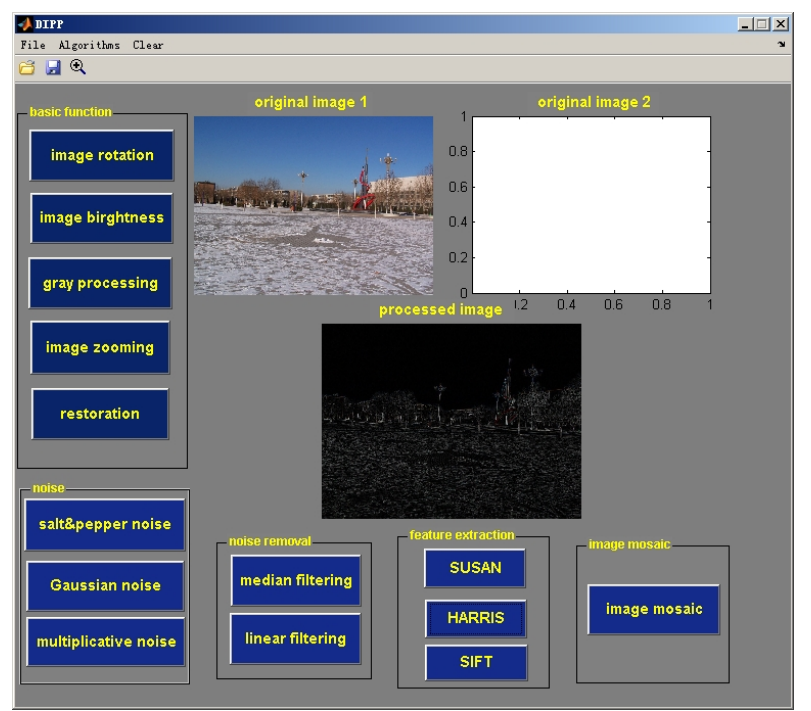

Figure 6. Feature extraction module function

Selecting the image mosaic module, we can realize the image mosaic function. Image mosaic can joint two images having overlapping region. Here the Harris corner detection method is used. Figure.7 shows the simulation result.

\section{Summary}

This paper presents the use of GUI tool for designing an image processing virtual simulation platform based on MATLAB. MATLAB provides an easy way to build GUI with elements such as radio buttons, check boxes, pushbuttons, list boxes etc., and link them to bulid-in MATLAB functions. 
We utilize these controls to integrate some basic image processing functions, such as, image rotation, image brighten, image graying, image zooming, noise adding, noise removal, feature extraction, and image mosaic. The platform realizes good interactivity and expansibility.

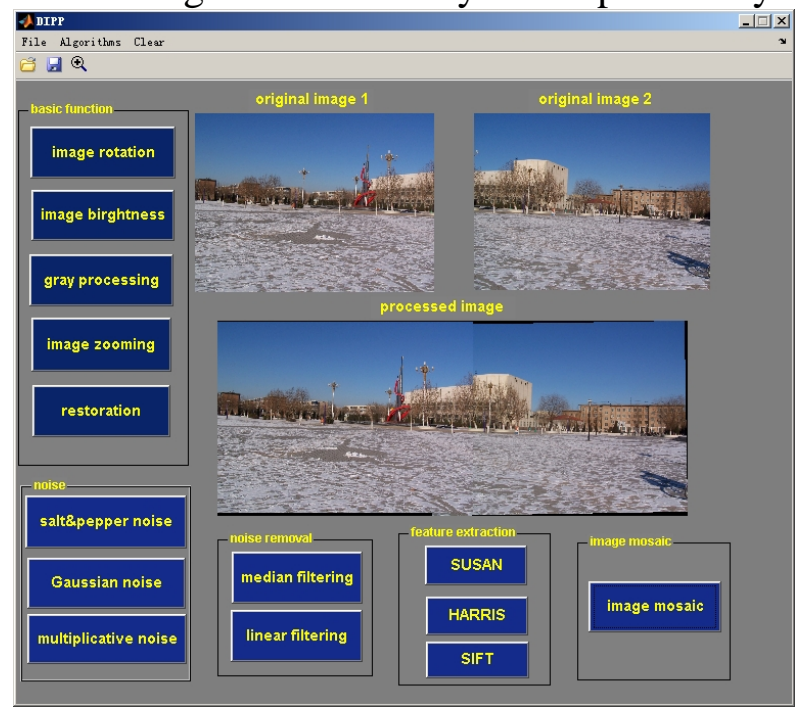

Figure7. Image mosaic module function

\section{Acknowledgement}

This research was financially supported by the Liaoning province Doctor Startup Foundation, China (Grant No. 201601213), and the Liaoning province College Students Innovation and Entrepreneurship Project, China (Grant No. 201611035000016).

\section{References}

[1] J.R. Raj, S.M.K. Rahman, S. Anand. Microcontroller USB interfacing with MATLAB GUI for low cost medical ultrasound scanners, Engineering Science and Technology, 2016, 19(2): 964-969.

[2] O. Tuzel, J. Thornton, J. Van Baar. Image denoising using a library of functions: U.S. Patent 9,262,810. (2016)

[3] P. L. Shui, W.C. Zhang. Corner detection and classification using anisotropic directional derivative representations. IEEE Transactions on Image Processing, 2013, 22(8): 3204-3218.

[4] S. Hu, Q. Wang, J. Wang, et al. Securing SIFT: privacy-preserving outsourcing computation of feature extractions over encrypted image data, IEEE Transactions on Image Processing, 2016, 25(7): 3411-3425.

[5] Q. Zhi, J.R. Cooperstock. Toward dynamic image mosaic generation with robustness to parallax. IEEE Transactions on Image Processing, 2012, 21(1): 366-378. 